\title{
RETRIEVING BROKEN INSTRUMENT IN HALF OF THE APIKAL ON CANINES TEETH WITH H-FILE BRAIDING TECHNIQUE
}

Yunita Styaningrum ${ }^{*}$ Yoanita Dwi Andina ${ }^{* *}$, Yulita Kristanti ${ }^{* * *}$

\author{
* Lecturer, Departement of Conservative Dentistry, Faculty of Dentistry, Universitas Islam Sultan Agung \\ ${ }^{*}$ Endodotist Practice, RSUD Caruban, Districts Madiun \\ ${ }^{* * *}$ Lecturer, Departement of Conservative Dentistry, Faculty of Dentistry, Universitas Gadjah Mada
}

Correspondence: yunitastyaningrum@unissula.ac.id

\section{Keywords:}

Failure in endodontic treatment; $\mathrm{H}$-file braiding technique; Retrieving broken instrument

\begin{abstract}
Background: Broken instruments affect the outcome and prognosis. A broken instrument will prevent the procedure of cleaning and shaping, irrigation and obturation of the root canal on the obstructed root section causing failure in endodontic treatment. There are some treatments for broken instrument including retrieving a broken fragment file and bypassing it on the root canal. the aim of the case report aims to provide an alternative for retrieving broken instruments using the $\mathrm{H}$-file braiding technique.

Method: A 38-year-old female patient came to the Dental Conservation Clinic RSGM Prof. Soedomo FKG UGM. A patient got a history of initial root canal treatment in a clinic, which ended with a separated file on canine teeth left upper. No complain pain, from the investigation radiographic there is an instrument broken in half apical. The teeth 23 seen temporary restoration cavity class I is still in good. Retrieving broken instruments using the $\mathrm{H}$-file braiding technique, preparation with step back techniques, obturation with warm vertical condensation technique and class I composite resin restorations with fiberreinforced retention.

Result: No complain pain after 2 weeks retrieving broken instrument.

Conclusion: The technique of the $\mathrm{H}$-file braiding technique is one technique to retrieve a broken instrument, this procedure is simple, cost-effective, harmless to the teeth and gives root canal sealing.
\end{abstract}

\section{INTRODUCTION}

The success of endodontics treatment depends on the results of the cleaning and shaping, however many kinds of difficulties and accidents unwanted procedural can occur. One example of a common procedural problem is a broken instrument inside the root canal. 1,2 The incidence of broken files is $1.68 \%-2.4 \%$ for rotary instruments and $0.25 \%$ for hand instruments. ${ }^{1}$ The various reasons that can cause a broken instrument in the root canal such as type of tooth and root canal anatomical variations like curved canals and accessory canals, over-instrumentation, increased speed with a rotary instrument, loss of tactile sensation with hand use instrument and improper filing techniques. ${ }^{3,4}$ The presence of broken files impedes the process of cleaning, forming and filling resulting in a poor prognosis and causing treatment failure. ${ }^{5,1}$ When broken instrument occurs, the operator has the choice of (1) bypassing and obturating the canal (2) leaving the instrument in the canal, or (3) retrieving the file segment either non-surgical or surgical.,3,6

The success rate for retrieval of fragment instruments in canal root to be between fifty-five 55\% and seventy-nine $79 \% .{ }^{7}$ Armamentarium and techniques for retrieving of file segment in root canals such as endodontic ultrasonic tips, GG Drills, masserann kit, roydent extractor, brasseler endo extractor, microtube retrieval methods, proultra endo, $\mathrm{H}$-file braiding technique and separated instrument removal system. 4,8,9 All techniques and 
armamentarium exhibit maximum efficacy and utility when used under magnification with either magnifying loupes or dental operating microscope. ${ }^{4}$

The file braiding technique involves the use of several $\mathrm{H}$ files. This method effective when the fragment position deeply in the canal and not visible and the operator depends on tactile sense or the fragment is loose but cannot be retrieved by using other means. ${ }^{4} \mathrm{H}$ - file braiding technique is useful in retrieving pieces of a fractured instrument from the root canal and in general a success rate of $55 \%$ has been reported. ${ }^{10}$ The aim of the case report aims to provide an alternative for retrieving broken instruments using the $\mathrm{H}$-file braiding technique.

\section{CASE REPORT}

A 38-year-old female patient came to the Dental Conservation Clinic RSGM Prof. Soedomo FKG UGM. A patient got a history of initial root canal treatment in a clinic, which ended with a separated file on canine teeth left upper. No complaint of pain and no history of systemic disease. The objective , the tooth was restored with temporary restoration cavity class I is still in good, percussion: (+), palpation: (-), mobility: 0 degree and probing mesial: $2,3 \mathrm{~mm}$. The radiographic examination there is an instrument broken in half apical, a radiolucent area at apical 23 and the radiolucent area in the interdental between 22 and 23 (figure 1).

The diagnosis is made of 23 teeth are profound caries, pulp necrosis, symptomatic apical periodontitis with instrument fragments in half of the apical. The treatment plan is retrieving fragment instrument with $\mathrm{H}$-file, endodontics treatment with step-back techniques and class I composite resin restorations with fiber-reinforced retention. The prognosis is Bonam because the remaining structure of tooth hard tissue 23 which can still be restored, the supporting tissue is still quite good, straight and large root canal, the location of the fractured instrument is affordable, no mobility, no history of systemic disease and cooperative patient.
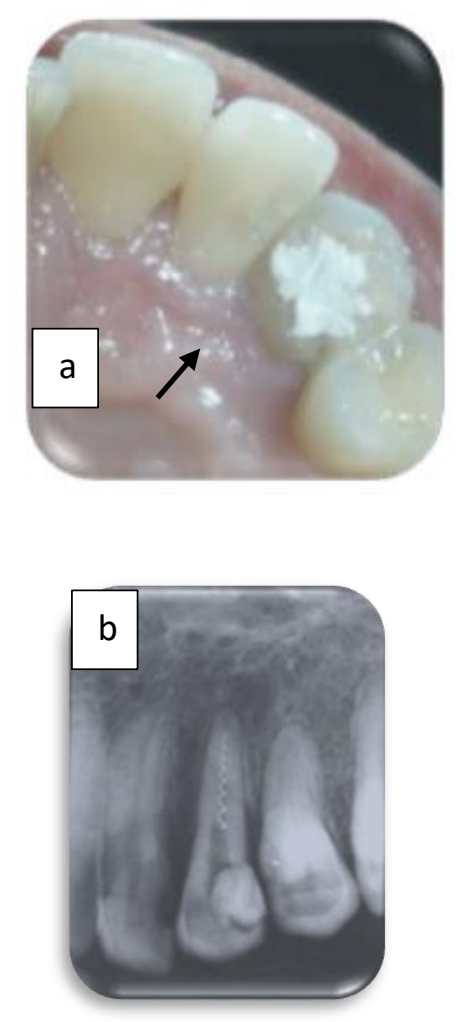

Figure 1. (a) a clinical of teeth 23 seen temporary restoration (b) Periapical radiograph show the presence of instrument fragments on the apical 1/2 teeth 23

In the first visit, the temporary filling was removed and the attempt was made to retrieve broken instruments in half of the apical. Radiographic examination appears that the file can pass through the instrument fragment until reaches apical so that there is a gap between the root canal walls is present and fragment (figure 2). The retrieved fragment, the root canal was irrigated with $2.5 \%$ sodium hypochlorite and $15 \%$ EDTA. The location of the broken file was half of the apical, Gates Glidden drills were used for coronal enlargement and then Two $\mathrm{H}$-files were inserted. One $\mathrm{H}$-files in buccal and the other in palatal, and then the files were braided in the clockwise 
direction, to engage the file segment inside the canal. The file segment came out of the canal along with the $\mathrm{H}$-files. A radiograph was taken to confirm the complete removal fragment file (figure 2).

The working length (WL) measurement with an apex locator (Dentsply) and confirmed by radiography obtained a working length of $25 \mathrm{~mm}$ (figure 3). Root canal preparation is done by using the step-back technique with IAF \#30. Starting with apical preparation, root canal body, coronal preparation and finishing. Every replacement of root canal file is carried out irrigation and recapitulation. Calcium hydroxide dressing is placed for seven days.
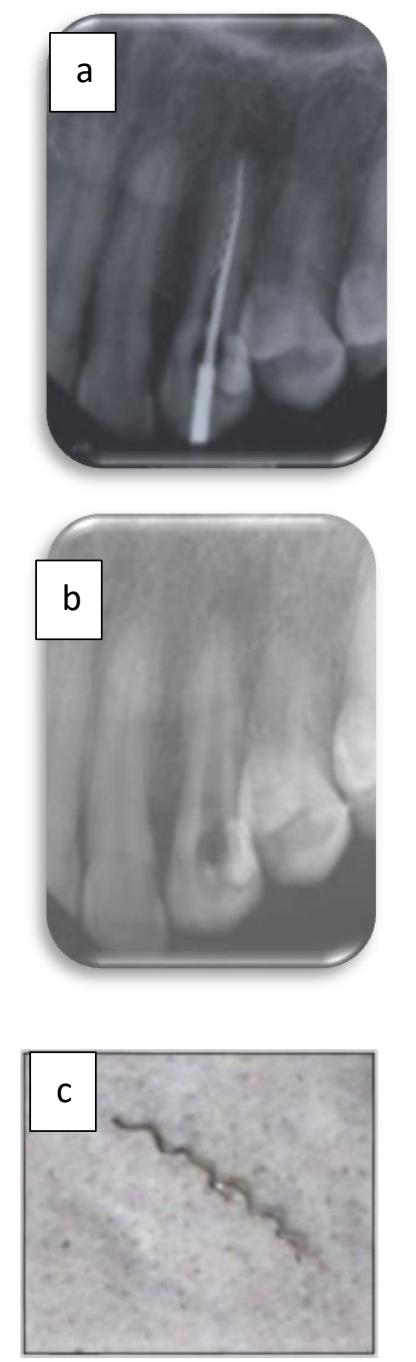

Figure 2. (a) Radiographic teeth 23, it appears that the file can pass through the instrument fragment until it reaches apical, (b) A radiograph confirm the complete removal fragment file, (c) fragment file.

In the second visit, removal of calcium hydroxide dressing and obturation was done using warm vertical condensation technique with guttapercha and sealer resin-modified glass ionomer (figure 3). The next step is to reduce gutta-percha 5 $\mathrm{mm}$ under the orifice and cavity preparation, class 1 preparation design and short bevel at cavosurface margin. Polyethylene fiber bands (Construct, Kerr) are inserted into the root canal for intracoronal retention (figure 4), class 1 composite resin restoration is carried out and restoration is done finishing and polishing (figure 5).

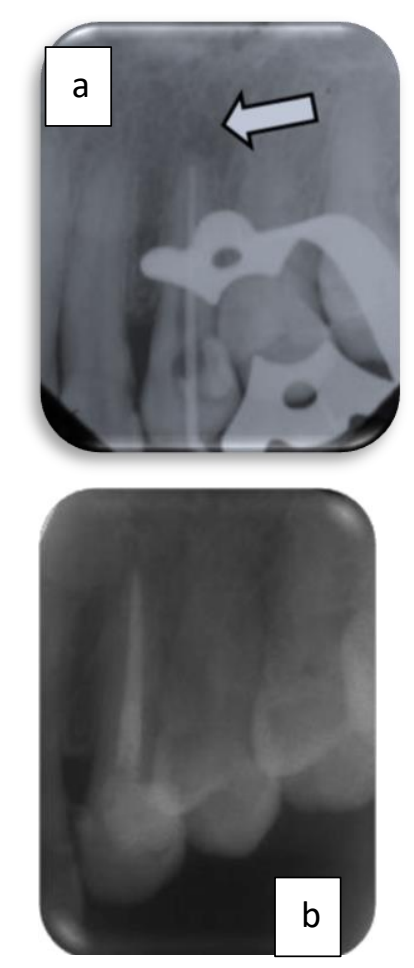

Figure 3. (a) A radiograph working length, (b) radiographic root canal obturation 


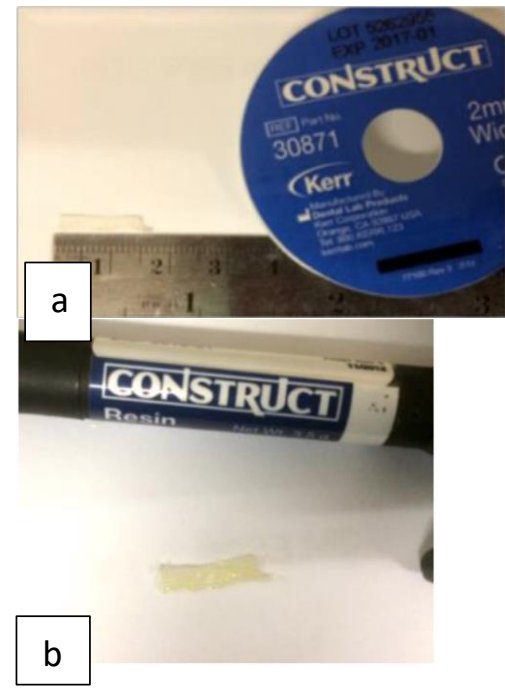

Figure 4. (a) Polyethylene fiber band (Construct, Kerr) width $2 \mathrm{~mm}$ length $1.5 \mathrm{~cm}$, (b) Polyethylene fiber band that has been smeared with resin (Construct Resin, Kerr).

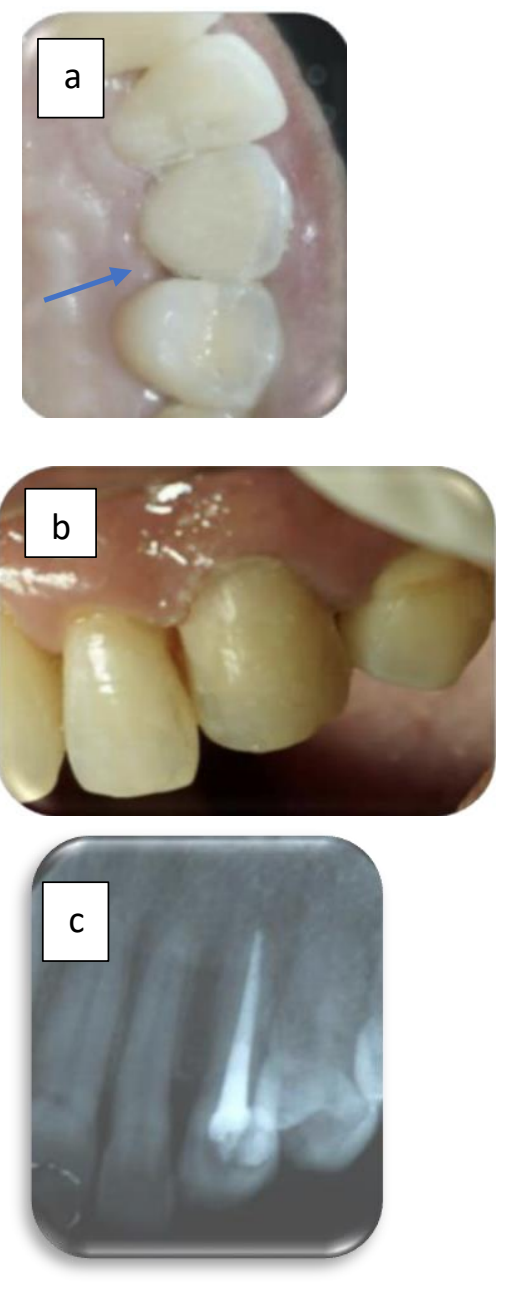

Figure 5. (a) Clinical overview postrestoration 23 of the palatal, (b) Overview clinical post-restoration facial appearance (c) Post-restoration periapical radiographs.

\section{DISCUSSION}

In the present case reports, we used a conservative approach with $\mathrm{H}$-file braiding technique to remove the file segment and to cause less harm to the tooth, by preserving the root canal dentin. ${ }^{3}$ Gates Gliden drills were used for coronal enlargement because important factors to be considered prior to instrument retrieval is to obtain a straight-line access to the coronal end of the separated instrument. 3,8 Two $\mathrm{H}$-files were put into the root canal under excessive irrigation of $15 \%$ EDTA and sodium hypochlorite. 15\% EDTA is a chelating agent that functions as a lubricant. ${ }^{10}$

The bypass technique is considered the safest treatment used in the case of a broken instrument file because it does not remove excessive amounts of dentin from the root canal. This technique reduces the contact between the instrument and the root canal dentin, as well as creates space for insertion of other instruments, such as ultrasonic tips or Terauchi kit, which can completely remove the fragment from the root canal. However, this technique is a very demanding technique where success depends on the sense of touch and persistence of the operator. Moreover, the success of this procedure depends on the ISO size and the taper of the broken instrument. the bypass technique takes time because it requires negotiation using hand files with small numbers to go through the files and reach the working length. In addition, during this technique, the wrong route of the negotiation file and the risk of perforation can occur. ${ }^{12,13}$ In this case, the operator chooses the $\mathrm{H}$ file Braiding technique, to retrieve files that are broken in the apical half because files can pass through broken file fragments and reach working lengths (figure 2), so the by-passing technique is not required in this case. 
The operator chose to use the custom post fiber technique because the prefabricated fiber post has optimal adaptation to teeth with small and circular root canals. In wide and irregular root canals as in the case, these posts are not indicated because they are poorly adapted and require a fairly thick resin cement. the retention and adaptation of

A success of file retrieval depends on the metallurgy of the broken file segment, canal anatomy, location of the fragment inside the canal, the length of the separated fragment, the diameter of the canal itself, and the plane in which the canal curves. ${ }^{3,1,11}$ Removal of the fractured fragment also

\section{CONCLUSION}

The technique of the $\mathrm{H}$-file braiding technique is one technique to retrieve a broken instrument, this procedure is simple, cost-effective, harmless to the teeth and gives root canal sealing.

\section{REFERENCES}

1. Meidyawati R, Suprastiwi E, Setiati HD. Case Report Broken File Retrieval in the Lower Right First Molar Using an Ultrasonic Instrument and Endodontic Micro Forceps. Hindawi. 2019;2019.

2. Subrata A, Hardini N. Removing a Fractured Instrument from the Root Canal Using Ultrasonic Tips. Sci Dent J. 2019;3(3):95-9.

3. Tomer AK, Muni S, Bharadwaj G, Malik N. Broken File Retrieval : Puzzle in Endodontics. Int J Oral Care Res. 2016;4(JanuaryMarch):75-8.

4. Mehta A, Bhagwat S, Kulkarni R, Padhya L. Retrievel of Separated Istrument From The Root Canal- A Review Of Techniques and Management of A Case. Int J Oral Heal Dent. 2018;4(2):130-235.

5. Gandevivala A, Parekh B, Poplai G, Sayed A. Surgical Removal of Fractured Endodontic Instrument in the Periapex of Mandibular First Molar. J Int Oral Heal. 2014;6(4 April):85-8.

6. RekhaGoyal D, ShikhaKanodia D, GirishParmar D, AbhishekParmar D. Alternative methods of Retrieval of separated Instruments - A Simpler Approach. J Gov Dent Coll Hosp. 2016;02(02 March):34-9.

7. Vyavahare N, Kulkarni M, Desai N. Retrieval of a Separated Nickel-titanium Instrument and a Stainless Steel $\mathrm{H}$-file Using a Novel the fiber post are improved by custom-made, to obtain a fiber post that adapts to the morphology of the root canal. In addition, the Fiber Construct Ribbon is a polyethylene fiber that has ultrahigh strength which is coated by plasma and reinforced by unfilled resin. ${ }^{14,15}$

becomes more predictable if a gap between the root canal walls is present and fragment and the total length of the separated instrument if more than onethird is exposed, the removal of the instrument becomes easy. ${ }^{7}$

\section{CONFLICT OF INTEREST}

No potential conflict of interest relevant to this article was reported.

Tube, Loop and File Technique. J Int Clin Dent Res Organ. 2018;10(10):88-92.

8. Natanasabapathy V, Sundar S, Koteeswaran V. Case Report Retrieval of fractured $\mathrm{Ni}$ - $\mathrm{Ti}$ rotary instrument using ultrasonics and file braiding technique under surgical operating microscope. Endodontology. 2017;29(1):658.

9. Nanhe S, Nanda Z, Reddy K, Agarwal AR, Murarka R, Patil N. Case Report Retrieval of A Separated Instrument Using Masserann Kit. J Appl Dent Med Sci. 2017;3(June):64-8.

10. Uddin MF, Alam MS, Howlader MMR, Moral AA. Retrieval Of A Fractured Instrument Using File Braiding Technique: A Case Report. Updat Dent Collage J. 2012;2(1):2530.

11. Alzahrani MS, Al-nazhan S. Retrieval of separated instruments using a combined method with a modified vista dental tip. Saudi Endod J. 2012;2(1):41-5 